\title{
POSTULATES FOR SPECIAL TYPES OF GROUPS
}

\section{BY RAYMOND GARVER*}

In defining a special type of group, such as commutative group or finite group, one may be content to add a suitable postulate, or possibly a number of postulates, describing the special property under consideration, to any set of postulates for a general group. It is, however, of interest to pursue the matter further, to determine whether a simplified set of postulates can be set up which will adequately describe the special type. A number of investigations of this sort have been made; the results of Weber, Huntington, and Hurwitz are especially worthy of note. $\dagger$

In the present paper I shall use as a basis the three-postulate definition of group which I recently presented in this Bulletin. $f$ Let there be given a set of elements $G(a, b, c, \cdots)$ and a rule of combination, which may be called multiplication, by which any two elements, whether they be the same or different, taken in a specified order, determine a unique result which may or may not be an element of $G$. This system forms a group if it satisfies the following three postulates:

I. If $a, b, c, a b, b c,(a b) c, a(b c)$ are all elements of $G$, then $(a b) c=a(b c)$.

II. If $a$ and $b$ are elements of $G$, there exists an element $x$ of $G$ such that $a x=b$.

III. If $a$ and $b$ are elements of $G$, there exists an element $y$ of $G$ such that $y a=b$.

The reader will recall that a familiar four-postulate definition of group employs these three postulates, and a closure postulate. That definition was due to Huntington and Moore and was, in

* As was reported in this Bulletin, vol.41, p. 781, the author of this paper died on November 7, 1935. He had not seen the proofs of this paper. THE EDITORs.

† Weber, Lehrbuch der Algebra, vol. 2, 1896, pp. 3-4; Huntington, Transactions of this Society, vol. 4 (1903), pp. 27-29, and vol. 6 (1905), pp. 22-24 and p. 186; Hurwitz, Annals of Mathematics, (2), vol. 8 (1907), p. 94, and vol. 15 (1913), pp. 93-94.

$\ddagger$ Vol. 40 (1934), pp. 698-701. 
turn, a simplification of Weber's set of eight postulates. It was of considerable interest to find that closure could be deduced from I, II, and III.

The questions which I wish to consider may now be outlined as follows. Let us write two more postulates:

IV. The number of elements of $G$ is finite.

V. If $a, b, a b, b a$ are all elements of $G$, then $a b=b a$.

We can clearly use postulates I, II, III, and IV to define a finite group, and postulates I, II, III, and V to define a commutative group. But is it now possible, in either case, to effect a simplification? I find that it is; in the case of the finite group a rather considerable part of one postulate proves to be redundant, while in the case of the commutative group three postulates may be combined to form one.

Let us first take up the case of the finite group. Consider the following postulate:

III'. There exists an element $g$ of $G$ such that, if $b$ is an element of $G$, there exists an element $y$ of $G$ such that $y g=b$.

I shall prove that I, II, III', and IV define a finite group. That this is really a substantial saving seems to follow from the fact that III postulates the solvability, in $G$, of $n^{2}$ equations, where $n$ is the number of elements of $G$, while III' postulates the solvability of but $n$ equations of the same form.

The proof will consist in deducing the closure property* from I, II, III', and IV, in fact, from II and IV only, and in then deducing III from I, II, III', IV, and closure. Note first that the deduction of closure from I, II, and III, as given in my earlier Bulletin paper, fails to carry through if we are working from I, II, and III'. However, a simple derivation can be obtained from II and IV. Consider the multiplication table of the group to be arranged as follows:

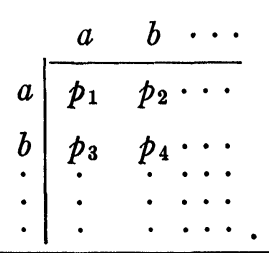

* If $a$ and $b$ are elements of $G$, the product $a b$ is an element of $G$. 
Thus, $a a=p_{1}, a b=p_{2}, \cdots$. By IV, the body of the table has $n$ rows and $n$ columns, but we do not know whether the products $p_{1}, p_{2}, \cdots$ occurring there are elements of $G$. Assume that the element $a$ of postulate II is temporarily fixed; say it is the $a$ of the table above. Then postulate II says, in effect, that no matter what element $b$ is, it will be found somewhere in the first row of the body of the table. In other words, the $n$ places in the first row must be filled by the $n$ elements in some order, and closure is satisfied as far as this first row is concerned. But the same argument shows that the $n$ places in each row of the multiplication table must be filled by the $n$ elements in some order, and closure is established. Obviously this simple argument is not valid when the number of elements is infinite.

We next require two lemmas.

Lemma 1. If $e, f$, and $g$ are elements of $G$ ( $g$ possessing the property described in $\left.\mathrm{III}^{\prime}\right)$ such that $e g=f g$, then $e=f$.

For otherwise the product $y g$, as $y$ represents in turn the $n$ elements of $G$, assumes fewer than $n$ distinct values. But this clearly contradicts postulate III'.

LEMмA 2. If e, $f$, and $d$ are elements of $G$ such that $e d=f d$, then $e=f$.

By II, there exists an element $x$ such that $d x=g$, where $g$ is an element possessing the property of III'. From $e d=f d$, we have $(e d) x=(f d) x$. By closure and I we may write $e(d x)=f(d \dot{x})$, or $e g=f g$. By Lemma $1, e=f$.

We have finally to exhibit an element $y$ which will satisfy III for an arbitrary choice of $a$ and $b$. By II, there exists an element $z$ such that $a z=g$, where $g$ again is an element of the type postulated in $\mathrm{III}^{\prime}$. By closure and $\mathrm{III}^{\prime}$, there is an element $y$ satisfying the equation $y g=b z$. For this $y$ we then have $y(a z)$ $=b z$, or $(y a) z=b z$. It follows from Lemma 2 that $y a=b$, and the deduction of III is complete.

It seems worth-while mentioning that a similar line of reasoning may be used to effect a simplification in Weber's set of postulates for finite groups. He employs closure, our postulates I and IV, and the following postulates.

VI. If $d, e$, and $f$ are elements of $G$ such that $e d=f d$, then $e=f$. VII. If $d, e$, and $f$ are elements of $G$ such that $d e=d f$, then $e=f$. 
Postulate VI has appeared in this paper as Lemma 2. We may replace it by the following one.

$\mathrm{VI}^{\prime}$. There exists an element $g$ such that eg $=f g$ implies $e=f$.

Closure and VII imply II, while closure and VI' imply III' Hence closure, I, IV, VI' , and VII define a finite group. Closure cannot be dispensed with in this set of postulates.

Passing now to commutative groups I find that, instead of using I, II, III, and V, we need only I and the following postulate.

VIII. If $a$ and $b$ are elements of $G$, there exists an element $x$ of $G$ such that $a x=x a=b$.

First, note that II and III are satisfied. To obtain V, we employ VIII and I and find $a b=a(x a)=(a x) a=b a$.

For purposes of comparison, I may add that Huntington's main definition of commutative group* employs a strengthened associative postulate, our postulate II, and a strengthened commutative postulate. His strengthened forms of I and V are as follows:

$\mathrm{I}^{\prime}$. If $a, b, c, a b, b c, a(b c)$ are all elements of $G$, then $(a b) c$ $=a(b c)$.

$\mathrm{V}^{\prime}$. If $a, b, b a$ are all elements of $G$, then $a b=b a$.

These are stronger than I and V, respectively, because their hypotheses are of wider range than those of I and V, while their conclusions are the same, respectively.

It is easy to show, on the basis of my Bulletin paper already referred to, that I may be substituted in Huntington's definition for $\mathrm{I}^{\prime}$. For if $a$ and $b$ are any two elements of $G$, there is an element $x$ such that $a x=b$, by II. By $\mathrm{V}^{\prime}, x a=a x=b$. Hence III is satisfied, and I, II, III, and $\mathrm{V}^{\prime}$ are certainly sufficient to define a commutative group. An interesting question remaining is whether the set of postulates I, II, and $\mathrm{V}^{\prime}$ may be replaced by I, II, and V. I have a feeling that this simplification is not possible, but I cannot give a proof at the present time. The reader will note, however, that III can no longer be deduced from II and $\mathrm{V}$ as it could from II and $\mathrm{V}^{\prime}$.

* Transactions of this Society, vol. 4 (1903), pp. 27-29. 
Hurwitz' definition of commutative group employs two postulates; ${ }^{*}$ the reader will find it interesting to compare with that of the present paper.

University of California at Los Angeles

\section{ON SOME QUADRATURE FORMULAS AND ON ALLIED THEOREMS ON TRIGONOMETRIC POLYNOMIALS}

\section{BY J. GERONIMUS}

1. Introduction. We consider the following problem.

Find $2 n$ numbers $0 \leqq \theta_{1}<\theta_{2}<\cdots<\theta_{2 n-1}<\theta_{2 n}<2 \pi$ such that for every trigonometric polynomial

(1) $\quad G(\theta)=\alpha_{n}+\sum_{k=0}^{n-1}\left\{\alpha_{k} \cos (n-k) \theta+\beta_{k} \sin (n-k) \theta\right\}$

of order $\leqq n$ the equality

$$
\int_{0}^{2 \pi} F(\theta) G(\theta) d \theta=L\left\{\sum_{i=1}^{n} G\left(\theta_{2 i-1}\right)-\sum_{i=1}^{n} G\left(\theta_{2 i}\right)\right\}
$$

holds true, where $F(\theta)$ is the given function

$$
F(\theta)=\sum_{k=n-s}^{\infty}\left(A_{k} \cos k \theta+B_{k} \sin k \theta\right), \quad(s \leqq(n-1) / 2),
$$

and $L$ is a given positive number.

Let

$$
\begin{aligned}
& F_{n}(\theta)=\sum_{k=n-s}^{n}\left\{A_{k} \cos k \theta+B_{k} \sin k \theta\right\} \\
& p_{n}(\theta)=-\int F_{n}(\theta) d \theta, \quad G^{*}(\theta)=\text { const. } \prod_{k=1}^{2 n} \sin \frac{\theta-\theta_{k}}{2} .
\end{aligned}
$$

Then, integrating (2), we get

\footnotetext{
* For references, see the first footnote to this paper.
} 